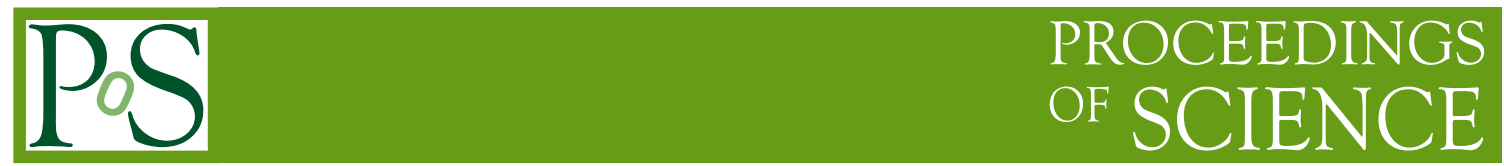

\title{
Neutrinos and the origin of the cosmic rays
}

\author{
Walter Winter* \\ DESY, Platanenallee 6, D-15745 Zeuthen, Germany \\ E-mail: walter.winter@desy.de
}

\begin{abstract}
We discuss the interplay between the high-energy neutrino flux observed by IceCube and cosmic ray observations. One question is if the neutrino flux can be reconciled with the paradigm that it comes from the sources of the UHECRs. Another one is how many of these neutrinos can stem from cosmic ray interactions with hydrogen in the Milky Way if the chemical composition of the cosmic rays is taken into account. Finally, we discuss if gamma-ray bursts (GRBs) can be the sources of the UHECRs in light of recent neutrino observations.
\end{abstract}

The 34th International Cosmic Ray Conference,

30 July- 6 August, 2015

The Hague, The Netherlands

${ }^{*}$ Speaker. 


\section{Introduction}

The recent discovery of a diffuse neutrino flux of presumably extra-galactic origin [1] has established neutrinos as one additional messenger in the field of high-energy astrophysics. Neutrinos may in particular offer a new perspective on the origin of the cosmic rays, as cosmic rays in the sources will interact with matter and radiation fields to produce neutrinos. Although the observed neutrinos originate from primaries at much lower energies, it is especially interesting if there can be a connection to the sources of the ultra-high energy cosmic rays (UHECRs). It is intriguing that the observed diffuse neutrino flux is at the level of the prediction from Waxman and Bahcall [2], which was estimated from the energy input required to power the UHECRs.

While cosmic rays are charged particles deflected by magnetic fields, neutrinos point back directly towards the source - at least theoretically. From the experimental point of view, neutrinos are detected in two event topologies: cascades (mostly from $v_{e}$ and $v_{\tau}$ ) and muon tracks (mostly from $v_{\mu}$ ). While cascades have better energy resolution, muon tracks allow for better directional reconstruction (of order one degree). Since the observed neutrino flux is dominated by electromagnetic and hadronic cascades because muon tracks are reconstructed at lower energies, the typical angular resolution for the observed events is only 10-15 degrees, sometimes even larger [1]. Future point source searches for the origin of the cosmic rays therefore have to rely on muon tracks.

The connection between neutrino/cosmic ray sources and gamma-ray sources is less evident, as in most cases radiation processes in the sources without cosmic ray loading can describe the gamma-ray observations. Nevertheless, the source of the gamma-rays can be often identified, and source catalogs are available for gamma-rays. Therefore, it is anticipated that at least a subset of the observed gamma-ray emitters, which imply radiation processes at relatively high energies, will lead to neutrino and cosmic ray emission. This assumption is the basis catalogue searches for the origin of the observed neutrinos. Although some hints for correlations of neutrino events with known gamma-ray sources exist, see e.g. [3], direct evidence cannot yet be claimed.

We therefore discuss the connection between neutrinos and cosmic rays from the conceptual point of view, focusing on three different questions: How many of the observed cosmic neutrinos come from cosmic ray interactions in the Milky Way? Can the observed neutrinos come from the sources of the ultra-high energy cosmic rays? Are GRBs the sources of the ultra-high energy cosmic rays?

\section{Neutrinos from cosmic ray interactions in the Milky Way}

As far as the origin of the observed diffuse neutrino flux is concerned, it is useful to first of all evaluate the "guaranteed" contributions, i.e., the contributions which we know that they should be present. One example are cosmogenic neutrinos from interactions between cosmic rays and the extra-galactic photon backgrounds, which are however expected to peak at much higher energies. Another example are contributions from cosmic ray interactions with gas in the Milky Way, see Refs. [6, 7, 8, 5, 9, 10].

First of all, it it noteworthy that the morphology of the signal (such as the expected solid angle window) can be inferred from gamma-ray observations [11] if a substantial fraction of these gamma-rays comes from the $\pi^{0}$ decays - where $\pi^{0} \mathrm{~s}$ are produced by these interactions as well. The 


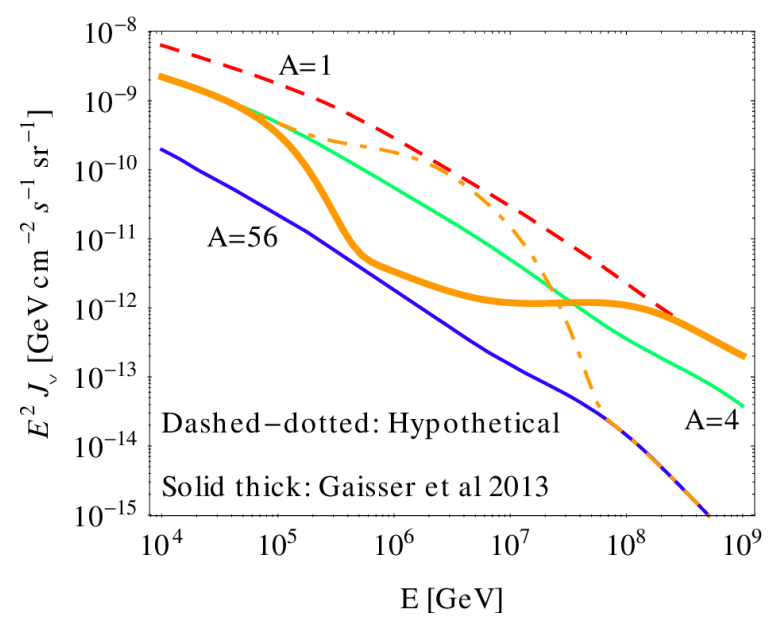

Figure 1: Predicted sky-averaged neutrino flux for (Galactic and extra-galactic) cosmic ray interactions with hydrogen in the Milky Way. Here $n_{H}=1 \mathrm{~cm}^{-3}$ and a cylindrical halo with radius $10 \mathrm{kpc}$ and half height $250 \mathrm{pc}\left(v_{\mu}+\bar{v}_{\mu}\right.$ flux including flavor mixing) have been assumed. The different curves are shown for different fixed mass numbers, a mono-nuclear composition following the compilation of data in Ref. [4], and a hypothetical composition change producing a peak at PeV energies. Figure taken from Ref. [5].

pion production will, however, depend on the cosmic ray distribution, density and composition, as well as the target gas density. It does not directly depend on the cosmic ray injection and escape time (however, the cosmic ray density can be derived from these).

The question of how many of the observed neutrino events can come from cosmic ray interaction in the Milky Way has been addressed in Ref. [5]. Compared to other approaches, the assumptions are simple: The cosmic ray density is assumed to be similar to the observed one (which can be directly obtained from the flux) everywhere in the Milky Way, and the cosmic ray composition is described by one component only. The corresponding all-sky averaged prediction is shown in Fig. 1 for different compositions, where the thick solid curve corresponds to Fig. 5, left, in Ref. [4] (the assumption of a single-component flux of iron leads to a pronounced dip). These are quite conservative assumptions, as the cosmic ray density may be higher close to the center of the Milky Way, and there may be sub-leading components in the cosmic ray "cocktail". These sub-leading components are common in Galactic cosmic ray models, as in Ref. [4] (see Fig. 4), and may dominate the secondary production (e.g. proton versus iron) even if the corresponding cosmic ray flux is lower. However, note that they are highly model-dependent, whereas our assumptions are solely based on observations. Another difference to many other approaches is that we take into account the interactions between extra-galactic cosmic rays and gas in our Galaxy as we use the observed cosmic ray flux directly, which means that the Galactic-extra-galactic transition is irrelevant for this approach. Therefore, there is no real cutoff in Fig. 1.

Fitting the expected neutrino flux to the observed IceCube data, taking into account the expected solid angle coverage of the events and the cosmic ray composition, and taking into account the current statistics increase to 54 events, out of which at most 20 possibly come from directions at least close to the Galactic plane, one can estimate using [5] that about 0.6 events (with large statistical errors) comes from cosmic ray interactions in our Galaxy. This estimate can be consid- 

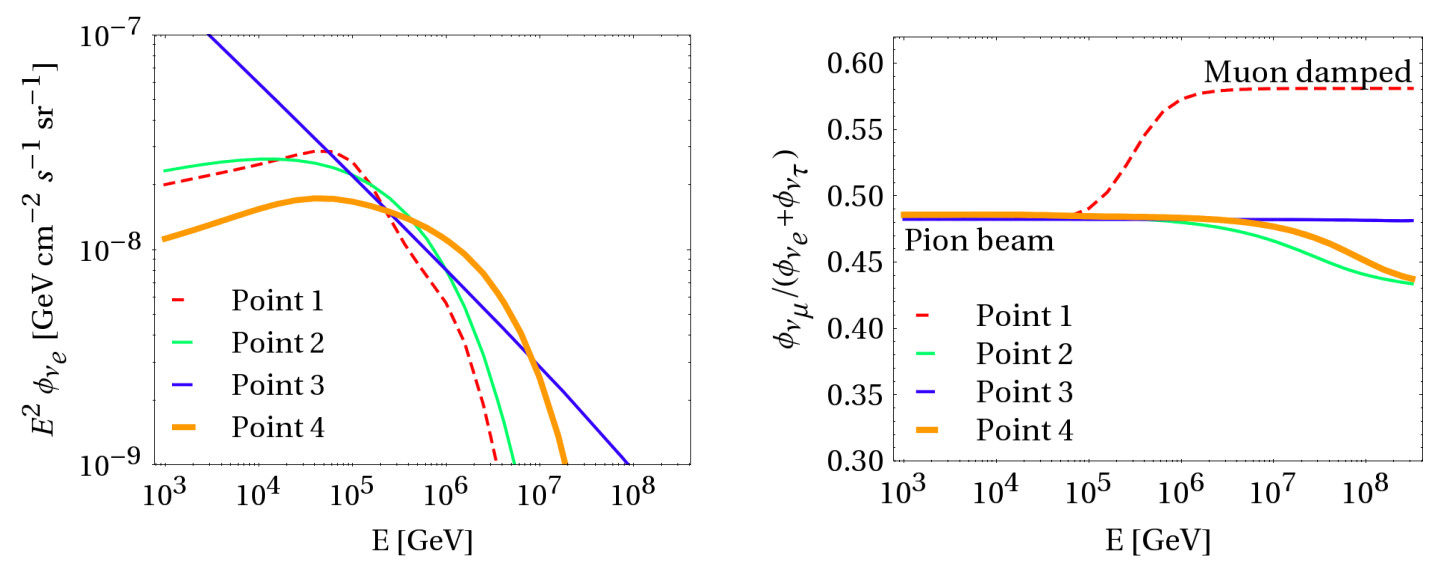

Figure 2: Spectra (left panel) and flavor composition at detector (right panel) for four different parameter sets (see main text) fitting the IceCube observed neutrino flux: Point 1 refers to $\alpha=-2, E_{\max }=10^{9} \mathrm{GeV}$, and $B=10^{4} \mathrm{G}$, point 2 to $\alpha=-2, E_{\max }=10^{7.5} \mathrm{GeV}$, and $B \lesssim 1 \mathrm{G}$, and point 3 to $\alpha=-2.5, E_{\max }=10^{11} \mathrm{GeV}$, and $B \lesssim 1 \mathrm{G}$. Point 4 refers a transition model into a heavier composition with $\alpha=-2, E_{\max }=10^{10.1} \mathrm{GeV}$, $B \lesssim 1$ G.Figure taken from Ref. [12].

ered as a minimum if the sub-leading components contribute, but it is a very robust expectation based on observations only. An upper limit estimate may be obtained using a hydrogen-dominated composition, leading to 3.2 events (with statistical errors). Therefore, cosmic ray interactions in our Galaxy cannot be the main component of the observed diffuse neutrino flux.

\section{Connection between observed neutrinos and UHECRs?}

Let us know discuss the question if the neutrinos can come from the sources of the UHECRs - which is legitimate since most neutrinos seem to be of extra-galactic origin. In order to address this question, we first of all identify possible ways how the data can be described. One possibility are photohadronic $(p \gamma)$ interactions, another one interactions of nuclei with matter $(A p)$.

The simplest case are $A p$ interactions, as the secondary spectra follow (in the absence of magnetic field effects) the primary spectrum of the protons or nuclei. In Ref. [12] four different ways were identified to describe the current data, see Fig. 2, left panel, where obviously the potential cutoff at $\mathrm{PeV}$ energies play an important role. The simplest possibility are proton interactions with a soft unbroken power law (spectral index of the primaries $\alpha \sim-2.5$, point 3 ), in consistency with Ref. [13]. This option however overproduces gamma-rays in contradiction with Fermi diffuse flux measurements [7]. If one wants to stick to $\alpha \simeq-2$, as expected from Fermi shock acceleration, one has the options of magnetic field effects on the secondary pions and muons (point 1) or a spectral break or cutoff (point 2). A similar effect can be produced if the composition turns into a heavier one with increasing energy (point 4). It is noteworthy that these options can be hardly distinguished in the relevant energy range (about $10 \mathrm{TeV}$ to $1 \mathrm{PeV}$ ). However, the point 1 with magnetic field effects leads to a transition from a pion beam to muon damped source in the cutoff region (see Ref. [14]), which can be in principle accessed by the ratio between muon tracks and cascades, see right panel in Fig. 2. 
Now which of these options can be reconciled with the paradigm that these neutrinos stem from the same sources as the UHECRs? This question is obviously non-trivial, as about three orders of magnitude in energy lie between the neutrino primaries and the UHECRs. It turns out that all of the above options work in conjecture with the UHECR paradigm, but all face challenges. Point 1 implies strong magnetic fields in the source, which also affect the proton maximal energy by synchrotron cooling. Therefore, high Doppler factors are required for this connection, which point towards a population of GRBs (low luminosity GRBs?) as potential candidate class. Point 2 implies a cutoff in the primary spectrum within the source, which need not be present in the escaping cosmic rays if the escape is energy-dependent. Candidate examples may be hypernova remnants or starburst galaxies. For point 3, the energy input per decade depends on energy, which leads to problems with the energy budget if extrapolated over several orders of magnitude, see e.g. Ref. [15] for a generic discussion and Ref. [16] for GRBs. Point 4 is challenged by a very shallow scaling of the composition with energy in contradiction with a pure rigidity scaling, which is difficult to reconcile with typical source models and maybe even the observed cosmic ray composition.

For photohadronic neutrino production, the secondary spectra will depend on the target photon spectrum as well. If the target photons are produced by synchrotron radiation of co-accelerated electrons as in AGNs, predictions can be made as a function of $R$ (size of the acceleration region) and $B$ (magnetic field), which are the Hillas parameters, see model in Ref. [17]. However, since the neutrino flux depends on the target photons, but not the cosmic ray flux, similar conclusions to the $A p$ model can be obtained, see Ref. [18]. Large proton energies to describe the UHECRs require large $R$ and relatively low $B$ (to avoid proton synchrotron cooling). The neutrino observations, however, prefer a cutoff, which can come from the maximal proton energy directly or from strong magnetic field effects on the secondaries. This leads to a tension between the parameter spaces fitting UHECR and neutrino observations, and indicates that it is possibly difficult to describe the observed neutrinos and UHECRs at the same time in "compact" objects, such as AGNs.

In summary, we have illustrated that the observed neutrinos may stem from the sources of the UHECRs. However, from the measured spectrum this connection is not obvious - and even imposes some challenges on the models.

\section{Neutrino-UHECR connection for Gamma-Ray Bursts}

GRBs are interesting for neutrino telescopes as a virtually background-free measurement is possible in stacking analyses due to the relatively short duration of the GRB. Using the gammaray information to predict the neutrino flux, current observations [21] reach the predictions, such as Ref. [23] - these predictions are, however, strongly parameter dependent. The constraints on GRBs as potential UHECR sources become more robust if cosmic rays escape as neutrons [24, 21]. However, it has been demonstrated in Ref. [16] that the UHECR escape mechanism is critical for this connection; two examples, normalized to UHECR observations, are shown in Fig. 3 for comparison. Note that the baryonic loading $\left(f_{e}^{-1}\right)$ is in these cases obtained from the fit of the data, i.e., it is not an input.

A further challenge has been addressed in Ref. [25]: efficient energy dissipation models for GRBs in the internal shock scenario imply a relatively broad range of collision radii. As a consequence, different messengers typically come from different production regions (see Fig. 4, left 

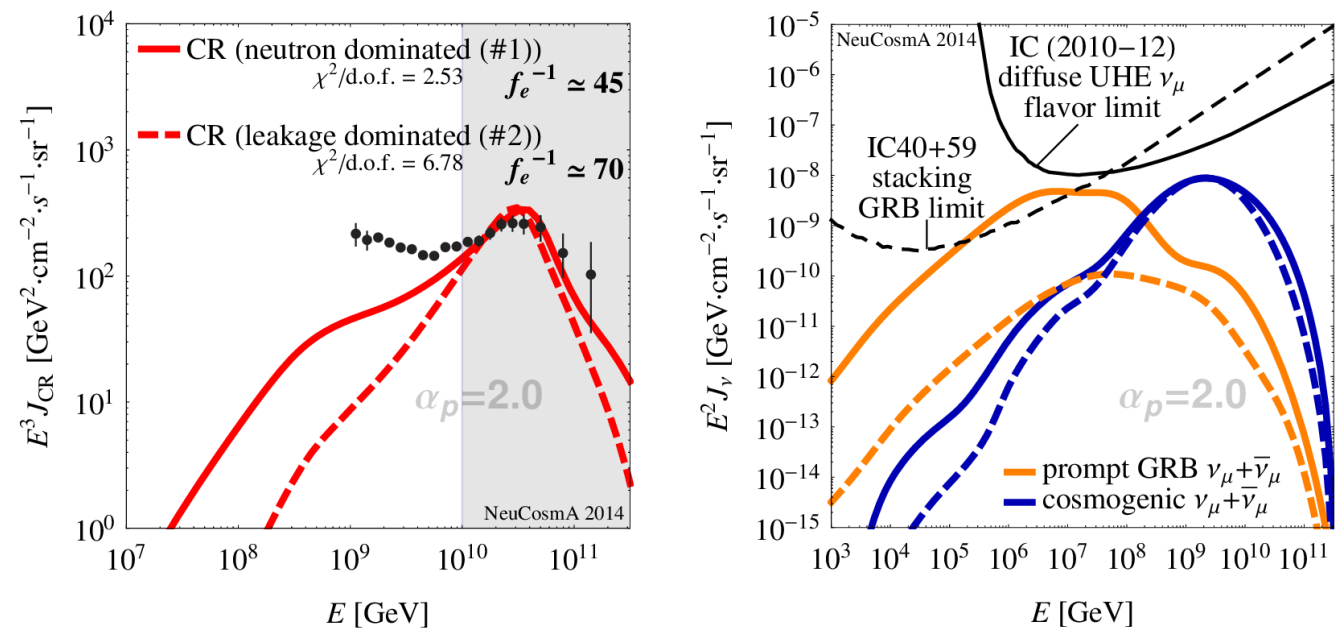

Figure 3: Best-fit cosmic ray (left panel) and resulting neutrino (right panel) fluxes as a function of energy for $\alpha=2$ and the Hopkins \& Beacom star formation rate [19]. The solid curves imply that neutron emission dominates the UHECR escape, the dashed curves mean that directly escaping protons dominate at the highest energies, where the Larmor radius reaches the width of the fireball shells. In the left panel, the observed UHECR data from the Telescope Array [20] are reproduced by the model (red curves) for two different parameter sets. Additionally, the fit range is gray-shaded, and the $\chi^{2} /$ d.o.f. and obtained baryonic loading $f_{e}^{-1}$ are given. In the right panel, the corresponding prompt $(\mathrm{PeV})$ and cosmogenic $(\mathrm{EeV})$ muon neutrino fluxes are given, together with the current bounds [21, 22]. Figure taken from Ref. [16].

panel) and the multi-messenger connection becomes more model-dependent than previously anticipated. Since the neutrino flux is dominated by collisions from close to the photosphere, a kind of minimal prediction for the neutrino flux can be derived which is comparatively robust with respect to the input parameters (thick solid curve in Fig. 4, right panel) - whereas the one zone prediction (dashed curve) strongly depends on the parameters. This flux may be actually enhanced by subphotospheric contribution (shaded area), which can however not be related to gamma-ray observations.

As a conclusion, GRBs can plausibly be the sources of the UHECRs - even if they may not describe the observed diffuse neutrino flux, and even if nuclei are accelerated to high energies [26].

\section{Summary and conclusions}

We have demonstrated that only few of the observed cosmic neutrinos can come from cosmic ray interactions in the Milky Way. Most of the neutrinos are (apart from atmospheric backgrounds) presumably of extra-galactic origin. The connection with the sources if the UHECRs is, however, less obvious, as it requires several orders of magnitude extrapolation in energy, as well as composition. In specific fit scenarios, there are generic consequences for the sources if this connection is to be implemented. Finally, GRBs may not be the dominant source of the observed diffuse neutrino flux, but can nevertheless be the sources of the UHECRs. A detection of neutrino events from GRBs may be the most spectacular test of an individual object class in the future. 

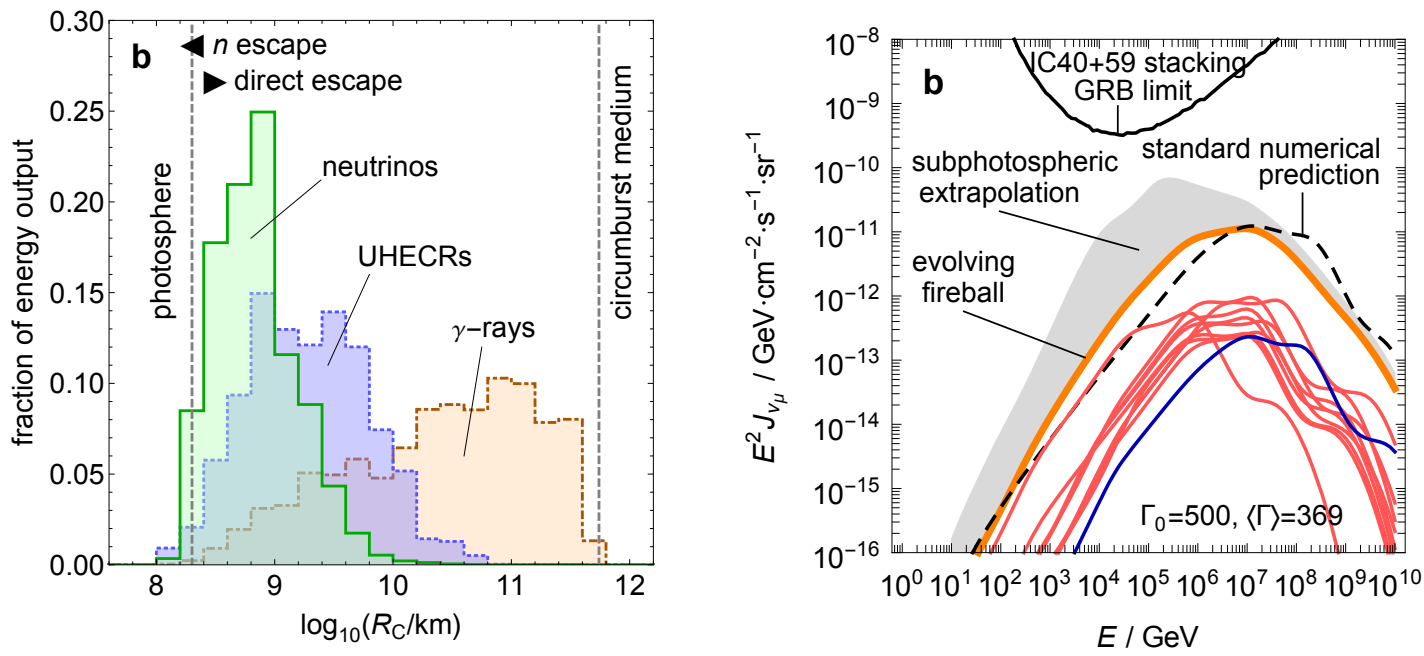

Figure 4: Left panel: Energy dissipated in (prompt) gamma rays, neutrinos (all flavors), and UHECR protons (from $10^{10}$ to $10^{12} \mathrm{GeV}$ ) beyond the photosphere. Here the fraction of energy output is normalized to one for each messenger. Right panel: Predicted quasi-diffuse neutrino spectra: Muon-neutrino spectrum from collisions beyond the photosphere (thick orange curves), reference spectrum computed from averaged burst parameters in the conventional one zone approximation (dashed curves), and maximal subphotospheric extrapolation (shaded regions). The individual (dominant) collisions (contributing to the thick orange curve) are shown also as thin curves. The diffuse GRB flux limit from the IC40+59 analysis [21] is shown as a thin black curve. Figure taken from Ref. [25].

\section{References}

[1] IceCube Collaboration, M. Aartsen et al., Evidence for High-Energy Extraterrestrial Neutrinos at the IceCube Detector, Science 342 (2013) 1242856, [arXiv : 1311.5238 ].

[2] E. Waxman and J. N. Bahcall, High energy neutrinos from astrophysical sources: An upper bound, Phys. Rev. D59 (1999) 023002, [hep-ph/9807282].

[3] M. Petropoulou, S. Dimitrakoudis, P. Padovani, A. Mastichiadis, and E. Resconi, Photohadronic origin of $\gamma$-ray BL Lac emission: implications for IceCube neutrinos, Mon.Not.Roy.Astron.Soc. 448 (2015), no. 3 2412-2429, [arXiv:1501.0711].

[4] T. K. Gaisser, T. Stanev, and S. Tilav, Cosmic Ray Energy Spectrum from Measurements of Air Showers, Front.Phys.China 8 (2013) 748-758, [arXiv: 1303.3565 ].

[5] J. C. Joshi, W. Winter, and N. Gupta, How Many of the Observed Neutrino Events Can Be Described by Cosmic Ray Interactions in the Milky Way?, Mon.Not.Roy.Astron.Soc. 439 (2014), no. 4 3414-3419, [arXiv:1310.5123].

[6] C. Evoli, D. Grasso, and L. Maccione, Diffuse Neutrino and Gamma-ray Emissions of the Galaxy above the TeV, JCAP 0706 (2007) 003, [astro-ph / 0701856 ].

[7] K. Murase, M. Ahlers, and B. C. Lacki, Testing the Hadronuclear Origin of PeV Neutrinos Observed with IceCube, Phys.Rev. D88 (2013), no. 12 121301, [arXiv: 1306.3417$].$ 
[8] A. Neronov, D. Semikoz, and C. Tchernin, PeV neutrinos from interactions of cosmic rays with the interstellar medium in the Galaxy, Phys.Rev. D89 (2014), no. 10 103002, [arXiv: 1307.2158 ].

[9] M. Kachelriess and S. Ostapchenko, Neutrino yield from Galactic cosmic rays, Phys.Rev. D90 (2014), no. 8 083002, [arXiv:1405.3797].

[10] M. Ahlers, Y. Bai, V. Barger, and R. Lu, Galactic TeV-PeV Neutrinos, arXiv: 1505.0315.

[11] Fermi-LAT Collaboration, Fermi-LAT Observations of the Diffuse Gamma-Ray Emission: Implications for Cosmic Rays and the Interstellar Medium, Astrophys.J. 750 (2012) 3, [arXiv:1202.4039].

[12] W. Winter, Describing the Observed Cosmic Neutrinos by Interactions of Nuclei with Matter, Phys.Rev. D90 (2014), no. 10 103003, [arXiv: 1407 . 7536].

[13] IceCube Collaboration, M. Aartsen et al., Atmospheric and astrophysical neutrinos above $1 \mathrm{TeV}$ interacting in IceCube, Phys.Rev. D91 (2015), no. 2 022001, [arXiv: 1410.1749 ].

[14] T. Kashti and E. Waxman, Flavoring astrophysical neutrinos: Flavor ratios depend on energy, Phys. Rev. Lett. 95 (2005) 181101, [astro-ph/0507599].

[15] B. Katz, E. Waxman, T. Thompson, and A. Loeb, The energy production rate density of cosmic rays in the local universe is $\sim 10^{44-45} \mathrm{erg} \mathrm{Mpc}^{-3} \mathrm{yr}^{-1}$ at all particle energies, arXiv : 1311.0287.

[16] P. Baerwald, M. Bustamante, and W. Winter, Are gamma-ray bursts the sources of ultra-high energy cosmic rays?, Astropart.Phys. 62 (2015) 66-91, [arXiv: 1401 . 1820].

[17] S. Hümmer, M. Maltoni, W. Winter, and C. Yaguna, Energy dependent neutrino flavor ratios from cosmic accelerators on the Hillas plot, Astropart. Phys. 34 (2010) 205-224, [arXiv: 1007.0006 ].

[18] W. Winter, Photohadronic Origin of the TeV-PeV Neutrinos Observed in IceCube, Phys.Rev. D88 (2013) 083007, [arXiv:1307.2793].

[19] A. M. Hopkins and J. F. Beacom, On the normalisation of the cosmic star formation history, Astrophys. J. 651 (2006) 142, [astro-ph/ 0601463 ].

[20] Telescope Array Collaboration, T. Abu-Zayyad et al., The Cosmic Ray Energy Spectrum Observed with the Surface Detector of the Telescope Array Experiment, Astrophys.J. 768 (2013) L1, [arXiv:1205.5067].

[21] IceCube Collaboration Collaboration, R. Abbasi et al., An absence of neutrinos associated with cosmic-ray acceleration in $\gamma$-ray bursts, Nature 484 (2012) 351-353, [arXiv: 1204 .4219].

[22] IceCube Collaboration Collaboration, M. Aartsen et al., First observation of PeV-energy neutrinos with IceCube, Phys.Rev.Lett. 111 (2013) 021103, [arXiv: 1304.5356 ].

[23] S. Hümmer, P. Baerwald, and W. Winter, Neutrino Emission from Gamma-Ray Burst Fireballs, Revised, Phys. Rev. Lett. 108 (2012) 231101, [arXiv:1112.1076].

[24] M. Ahlers, M. Gonzalez-Garcia, and F. Halzen, GRBs on probation: testing the UHE CR paradigm with IceCube, Astropart. Phys. 35 (2011) 87-94, [arXiv:1103.3421].

[25] M. Bustamante, P. Baerwald, K. Murase, and W. Winter, Neutrino and cosmic-ray emission from multiple internal shocks in gamma-ray bursts, Nature Commun. 6 (2015) 6783, [arXiv:1409.2874].

[26] N. Globus, D. Allard, R. Mochkovitch, and E. Parizot, UHECR acceleration at GRB internal shocks, arXiv:1409.1271. 